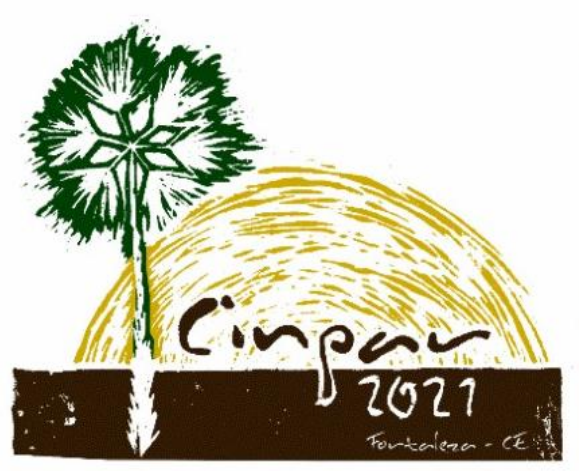

XVII Congresso Internacional sobre Patologia e Reabilitação das Construções

XVII Congreso Internacional sobre Patología y Rehabilitación de las Construcciones

XVII International Conference on Pathology and Constructions Rehabilitation

FORTALEZA (Brasil), 3 a 5 de junho de 2021

https://doi.org/10.4322/CINPAR.2021.080

\title{
HBIM - MODELAGEM DE INFORMAÇÃO PARA PRESERVAÇÃO DE PATRIMÔNIO
}

\author{
Abner ALMEIDA ${ }^{1}$, Leonardo INOJOSA ${ }^{2}$ \\ ${ }^{1}$ Faculdade de Tecnologia, Dep. De Engenharia Civil e Ambiental - UnB, Brasília, Brasil, abner_sa@hotmail.com.br \\ ${ }^{2}$ Faculdade de Tecnologia, Dep. De Engenharia Civil e Ambiental - UnB, Brasília, Brasil, leinojosa@gmail.com
}

\begin{abstract}
Resumo: Esse trabalho estudou a importância da Modelagem da Informação da Construção (BIM) para registrar e salvaguardar aspectos arquitônicos, históricos, de materiais utilizados, inúmeros outros, relacionados ao patrimônio edificado, visando à catalogação e inventátio para que possa ser utilizados em manutenções, restaurações e demais demandas. Exibiu um estudo bibliográfico sobre Historical Building Information Modeling (HBIM), que avalia e discute de modo crítico a utilização de tecnologias BIM a fim de garantir o gerenciamento da conservação, como também o suporte dos dados de manutenção e informacionais construtivos da edificação. A escolha da pesquisa foi delimitada pela necessidade visível de catalogação e documentação das obras históricas, visto que, quando catalogamos algo do gênero, eternizamos cultura, tradições e o legado da época que não se sabe se será perdido ou não por eventualidades. Enxergando essa realidade, vê-se como motivação para essa pesquisa o possível uso da ferramenta BIM com o objetivo de manter esse legado. Percebeu-se a importância da Modelagem da Informação da Construção (BIM) visanso à catalogação e inventários de dados e informações relacionadas com edificações do patrimônio histórico e cultural, visando a sua conservação.
\end{abstract}

Palavras-chave: Modelagem da Informação da Construção (BIM). Historical Building Information Modeling (HBIM). Conservação do Patrimônio Edificado

\section{Introdução}

O patrimônio histórico tem reconhecimento cultural precioso que necessita de preservação, as tecnologias digitais aparecem nesse cenário com uma funcionalidade imprescindível de auxílio na preservação do patrimônio arquitetônico. $\mathrm{O}$ uso de recursos e metodologias para reconstruir virtualmente uma edificação de âmbito histórico levanta probabilidades inovadoras para conservação da memória do edifício, como para sua manutenção e operacionalização.

A Modelagem da Informação da Construção (BIM) consiste em um recurso tecnológico de modelagem conexa a uma conjuntura de procedimentos de análise, comunicação e produção de padrões de construção BIM. Este também pode se determinar como uma conjuntura de aspectos processuais, e tecnológicos, sendo que há interação entre esses aspectos, causando um processo essencial de gerenciamento de informações do projeto na forma digital no decorrer da vida útil do edifício.

A utilização de tecnologias que agem na plataforma BIM admite o ajuste de padrões virtuais com dados informacionais estabilizados que, de outro modo, não teriam possibilidade de se integrarem em um singular apoio de dados. Os padrões BIM têm potencialidade para serem empregados na gerência da edificação, operacionalizando, realizando manutenção, reformando, requalificando e registrando edificações para finalidade de documentação arquitetônica.

O uso da documentação durante a etapa de operacionalização fornece informação e é uma forma de acompanhar, proativamente, as circunstâncias das edificações e instalações. A utilização do BIM se alargou para além do gerenciamento de edificações novas, atualmente, concebe a possibilidade de documentar e 
gerenciar a conservação de patrimônio de modo preciso e eficaz.

Justifica-se essa pesquisa pelo fato de o BIM ter potencialidade de disseminação de saberes referentes ao patrimônio edificado.

\section{Patrimônio Histórico}

De acordo com Fonseca (1997), foi em 1920 que o assunto do patrimônio começa a ser abordado no Brasil, sendo visto como basilar sob a ótica política, por meio do ato de proteger monumentos e artefatos de valor. O olhar para esse fator se deu por causa do estado ruim de conservação dos elementos históriconacionais, suscitando debates entre estudiosos e pesquisadores da época em meios comunicacionais e em entidades do governo.

O tombamento preserva as bases da história e da cultura a fim de que gerações usufruam das produções de artes passadas. Assim, percebe-se a relevância desse mecanismo de intervenção estatal, que conserva o patrimônio cultural e garante a satisfação do interesse coletivo, impedindo a perda de um patrimônio que configura a memória de grupos sociais.

A prevalência da estética e o dualismo estético-histórico monumental devem ser o cerne na conceituação de restauração de uma obra, enfatizando o valor artístico. Levando esse fator em consideração, o autor elenca três aspectos de diretrizes basilares para intervenção no monumento: Nenhuma intervenção de reparo pode impossibilitar operações posteriores, isso se refere ao parâmetro da reversibilidade; A operacionalização necessitará ser reconhecível, não podendo perder a unicidade da obra, isso se refere ao parâmetro da distinguibilidade (BRANDI, 2004). O objeto é insubstituível quando contribuir, de forma direta, para o aspecto figurativo da representação, não se limitando a questões meramente estruturais.

Em conformidade com Cunha (2010), esses parâmetros são hodiernos, pois se remetem à restauração com um ato atual a favor de gerações futuras. Reconhecer o valor cultural de um bem acarreta a obrigação de mantê-lo e de perpetuá-lo para próximas gerações, disponibilizando métodos e saberes sobre ele para que se preserve sua memória. A significação atualizada de bem patrimonial como sendo bem cultural expande seu espaço e, como consequência, a listagem de elementos a serem pesquisados e analisados, assim sendo, os debates e colóquios sobre ação e intervenção precisam acontecer.

\subsection{Preservação do patrimônio histórico}

A arquitetura moderna consiste em elemento complementar, estimada como a ampla proporção de edifícios dos locais contemporâneos, entretanto, por conta do seu pouco tempo de existência, não é reconhecida, pelo meio social, sua característica cultural de patrimônio que precisa ser eternizado para as gerações futuras. Assim sendo, há bastantes modelos de edifícios que correm risco de serem descaracterizados. Determinados atributos do movimento atual como a confiança no porvindouro e avanço e o indeferimento do acontecido, além das questões transitórias e efêmeras, não são justificativas para a não conservação dos elementos originários de obras. Em conformidade com Jukka Jokilehto (2003), a modernidade significa expressão cultural, deste modo consiste em um legado do século 20 a ser preservado.

A preservação, de acordo com Ghirardello e Spisso (2008), consiste na sustentação de um bem e no retardamento de sua deterioração, com a finalidade de resguardar o patrimônio cultural. Na maioria dos casos, o ato de preservar um bem é compreendido de maneira equivocada, por vezes errada, limitando-o à prática de conservação apenas. Em conformidade com o IPHAN (Instituto do Patrimônio Histórico e Artístico Nacional), a preservação é definida gestão prática do patrimônio a partir de mecanismos que identificam, protegem e gerenciam.

A Conferência em âmbito internacional de Conservação dos Monumentos Históricos, feita em Atenas em meados de 1930, originou a Carta de Atenas, de acordo com Cogima (2019), objetivando o estabelecimento de diretrizes e princípios universais para conservar e renovar monumentos históricos, devendo-se também, observar a valorização do entorno, como a fisionomia das cidades, preservação de algumas características pitorescas, planejamento paisagístico, materiais da restauração e ações diante da deterioração dos monumentos (COGIMA, 2019, p. 7). 


\section{BIM}

\subsection{Conceitos e Usos}

A National Building Information Modeling Standard (NBIMS) entende o BIM como ferramenta, produto e processo. No primeiro entendimento, refere-se a criar, agregar e extrair dados informacionais da amostra da edificação por meio de softwares; no segundo, apresenta- se o procedimento do projeto criado por mecanismos da tecnologia da informação, evidenciando o modelo da edificação; no terceiro, exibe-se um procedimento colaborativo de maneira que dados precisos para práticas cíclicas da edificação são obtidos.

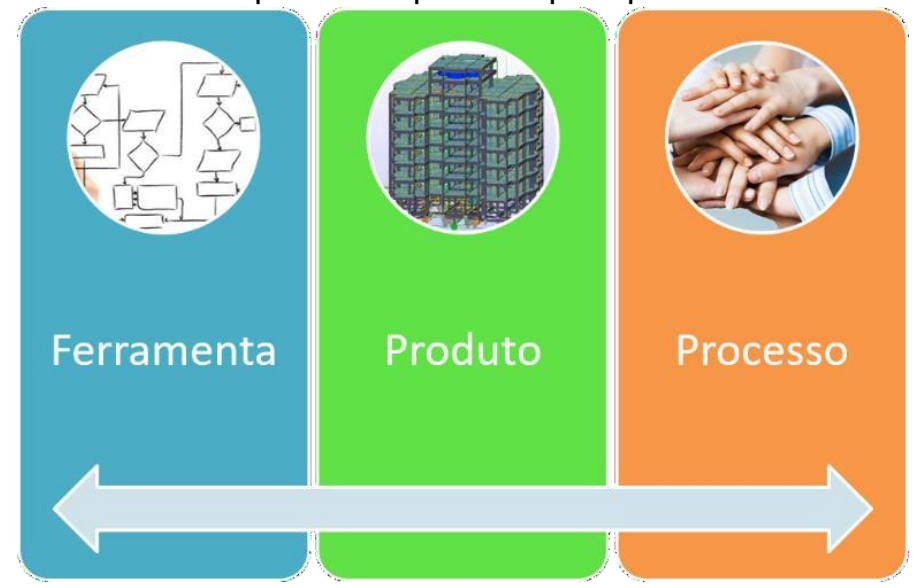

Figura 1 - BIM como Ferramenta, Produto e Precesso Fonte: Produzido pelo autor

O BIM (Building Information Modeling), em conformidade com Freitas (2019), existe a mais ou menos três décadas e, mesmo não sendo algo novo, é tido como um procedimento recente quando o é comparado com saberes da engenharia civil, que foram viáveis com o desenvolvimento tecnológico, disseminando-se depois da ampliação da demanda por computadores com desenvoltura boa de processamento e com valores viáveis para os negócios da construção civil.

\subsection{Nível de Desenvolvimento para Construção de Modelos de Informações (LOD) e Nível de Detalhe (LOD)}

Com base na criação de conceitos em 2008 e atualização em 2013 pelo Instituto Americano de Arquitetos, o Fórum BIM de 2013 disponibilizou uma documentação intitulada Especificação de Nível de Desenvolvimento para Construção de Modelos de Informações (LOD) que determina níveis: LOD 100, LOD 200, LOD 300, LOD 350 e LOD 400.

O primeiro é a apresentação simbólica ou de representatividade genérica, não consistindo em representatividade geométrica. Os dados informacionais desse nível são considerados aproximados ao que de fato existe. O segundo representa de forma gráfica um objeto ou uma sistemática genérica, com números, tamanho, formato, localização e direcionamentos aproximados.

O terceiro representa os componentes de modo preciso e dados não gráficos podem ser anexados também. No quarto nível, a representação dos elementos se dá de modo preciso quando se trata de quantitativo, dimensão, formato, localidade, direcionamento e interface com outras sistemáticas, dados que são aferidos de forma direta no modelo. No quinto nível, aparecem componentes expresso de forma gráfica precisa e detalhadamente, objetivando a fabricação.

O LOD (Level of Detail - Nível de Detalhe) consiste em um método, dentro da computação gráfica, que possibilita vários modos de representação para um mesmo item. Tal representação é operacionalizada em conformidade com as distintas distâncias possíveis. Nível de Desenvolvimento (Level of Development LOD) e Nível de Detalhe (Level of Detail - LOD) são distintos, ou seja, não são equivalentes.

O nível de desenvolvimento se refere ao grau de maturação e de credibilidade da implementação do modelo BIM, o nível de detalhe faz referência ao quantitativo de detalhes incluídos nos componentes do modelo. No Brasil, aplicar o mecanismo HBIM tem como propósitos a modelagem geométrica; a incorporação de materiais, métodos construtivos e informações de naturezas distintas; e o fornecimento de ferramentas para o gerenciamento do patrimônio histórico. Essas informações poderão, em momento oportuno, ser base de planos de intervenção, isso colaborará para a preservação eficiente do Patrimônio 
Histórico brasileiro. "A adoção da modelagem BIM no Brasil ainda se encontra em estágios iniciais, tanto no mercado da construção civil, quanto nos cursos de formação profissional em nível tecnológico, de graduação ou pós-graduação" (FREITAS, 2019, p. 40).

\subsection{HBIM - Conceito e Características}

Viabilizar a aplicação do HBIM ao patrimônio histórico é desafiante para gerenciar e valorizar os patrimônios. O desafio básico consiste na modelagem, visto que é preciso criar metodologias claras e simples para conseguir parâmetros que sejam precisos e de qualidade representativa. $O$ padrão necessita de informações que não se delimitam apenas em questões geométricas, também abrange dados históricos, exame de deterioração, dentre outros fatores.

O HBIM (Historic Building Information Modeling) consiste em um modo novo de criar modelos de edifícios que já existem fazendo uso do método BIM, possibilita a criação de padrões inteligentes que contenham e gerenciem informações. Esses padrões fazem referência a todos os elementos do projeto, abrangendo dados geométricos, físicos e de identidade de suas próprias partes.

Os primeiros usos do modelo BIM foram realizados em lugares de importância histórica e monumental. Pretendia-se criar um banco documental, em que haveria o modelo criado, buscando a introdução, adicionamento e compartilhamento de informações para várias utilidades como aspectos geométricos de volume e exames térmicos.

Logo, o HBIM consiste em um procedimento que se aplica a edifícios que já existem, podendo ser monumentais ou construções simplificadas de interesse histórico, que intensifica de forma considerável a potência do método BIM. O HBIM cria modelos de edifícios já existentes, indo além do desenho digital e geométrico 3D, dispondo de padrão inteligente que fornece informações. O HBIM determina um método novo de trabalho, e não uma nova tipologia de projeto. Esse procedimento possibilita a projeção do edifício existente elaborando um modelo do próprio edifício, apresentando a maior parte das suas especificidades de maneira mais real possível.

O HBIM direciona os edifícios existentes à engenharia reversa (reverse engineering): quando o edifício é modelado, há avaliação dos processos que possibilitaram a sua concretização, visando melhorar a modelagem. Normalmente, o modelo HBIM inicia por meio digital, com instrumentos como scanner a laser, permitindo a obtenção de nuvens de pontos - conjuntura de pontos conectados no espaço -, esses pontos têm dados geométricos, ou seja, coordenadas sistêmicas, e dados colorimétricos, que é a associação do ponto a cor que consta na fotografia.

O termo engenharia reversa tem suas origens no mundo do hardware. Uma empresa desmontar um produto de hardware competitivo na tentativa de conhecer os "segredos" de projeto e fabricação do concorrente. Os segredos poderiam ser facilmente entendidos se fosse possível obter as especificações de projeto e fabricação do concorrente. Mas esses documentos são de propriedade privada e não estão disponíveis para a empresa que está fazendo a engenharia reversa. Essencialmente, uma engenharia reversa bem-sucedida resulta em uma especificação de projeto e fabricação para um produto pelo exame de amostras atuais do produto (PRESSMAN, 2011, p.699 Apud Barbosa e Candido 2017).

$O$ método objetiva alcançar modelos BIM já existentes, firmando-se em elementos inteligíveis que abrangem dados informacionais de todo tipo, podendo ser atualizados, substituídos e enriquecidos. Esses elementos podem ser utilizados em edifícios parecidos, visto que aqueles são paramétricos, têm aspectos modificáveis e adaptáveis a situações específicas. Apesar ainda do pouco conhecimento sobre o HBIM por uma grande parte dos profissionais, os que têm ciência da sua imprescindibilidade no contexto atual de mundo e de valorização do patrimônio cultural buscam propagá-lo a partir da criação de banco de dados de componentes de edifícios, subsídios de estilos arquitetônicos. Sabe-se que por uma nuvem de pontos com resolução determinada há possibilidade de criação de modelo inteligível e paramétrico que abrange maiores informações sobre o edifício.

O procedimento tem um tempo demorado para ser terminado, visto que a maioria dos instrumentos BIM foram elaborados para novos projetos, e não para já existentes. Dessa forma, estudiosos analisam novas concepções e novos processos para que haja mais rapidez e automatismo na ação que, por meio da nuvem de pontos, alcança o modelo de um edifício, sem perder dados e detalhes.

Os fins que o HBIM pretende alcançar direcionam-se a estratégias, de acordo com o direcionamento do 
setor construtivo, que permitam gerenciar conhecimentos e elementos de modo coordenado entre os profissionais, como também gerenciar de forma eficaz e eficiente custos e recursos.

Fazer o cadastramento de um bem cultural tem como objetivo preservar os fatores imagéticos e históricos deste. A maioria dos bens históricos do Brasil ainda não foram documentados de modo devido. Diante da falta de uma sistemática cadastral e de um gerenciamento do patrimônio arquitetônico que use de modo adequado as tecnologias digitais, indica-se o emprego do HBIM (Historic Building Information Modeling), objetivando agilizar o procedimento de documentação, gerenciar de modo eficaz os bens e preservar de modo efetivo o patrimônio arquitetônico.

O HBIM se determina como expansão conceitual do BIM voltando-se para os aspectos históricos e peculiares de edificações. O Building Information Modeling (BIM) consiste em um método avançado de atividade colaborativa que utiliza o padrão elaborado a partir de dados seguros e coordenados. Enquanto os mecanismos CAD se centram em projetar e representar a edificação apenas, o padrão BIM almeja abranger o circuito de existência da edificação por inteiro, permitindo este cadastrar, estudar, acompanhar e manter o patrimônio arquitetônico, de modo que tal patrimônio se perpetue para gerações futuras.

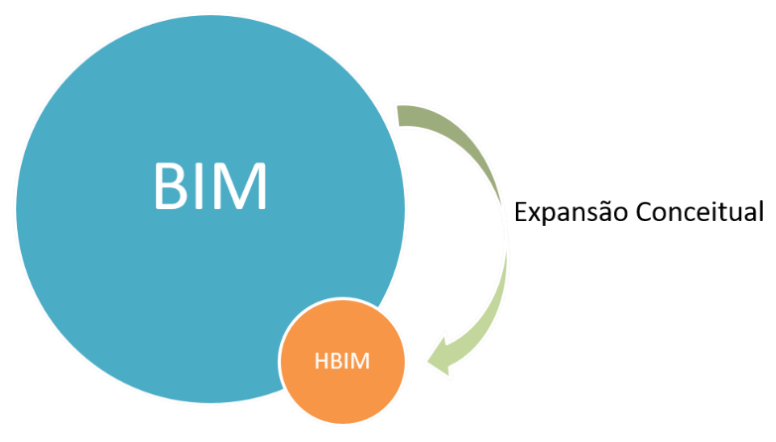

Figura 2 - HBIM como expansão Conceitual do BIM Fonte: Produzido pelo autor

Consoante Dore e Murphy (2012), o HBIM abarca recurso de engenharia reversa em que se realiza levantamento dos componentes arquitetônicos a partir da utilização de fotogrametria e/ou laser scanner. Depois de as informações levantadas são casadas a elementos paramétricos determinando a amostra finalizada da edificação.

\subsection{Uso HBIM}

O uso do HBIM consiste em uma manifestação geométrica 3D finalizada, compreendendo os pormenores do objeto como elementos e procedimentos construtivos. Em seguida, os dados levantados são combinados a objetos paramétricos, gerando o modelo completo da edificação. O produto final HBIM é um modelo geométrico 3D completo, incluindo detalhes do objeto como materiais e métodos construtivos. Então, probabilidades, projetações ortográficas, divisões, pormenores, cortes têm possibilidades de serem determinados com base na apreciação e na conservação de componentes históricos, composições e espaços.

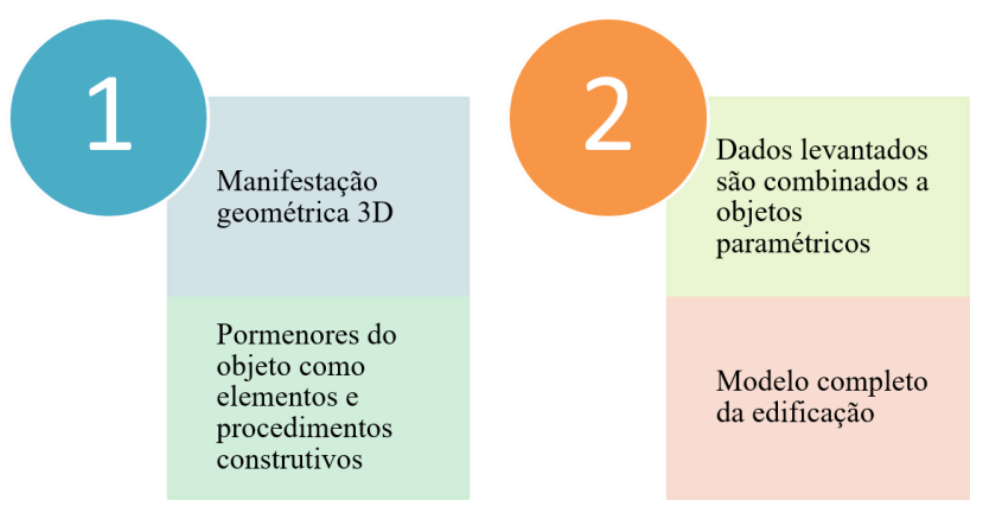

Figura 3 - Uso HBIM Fonte: Produzido pelo autor 


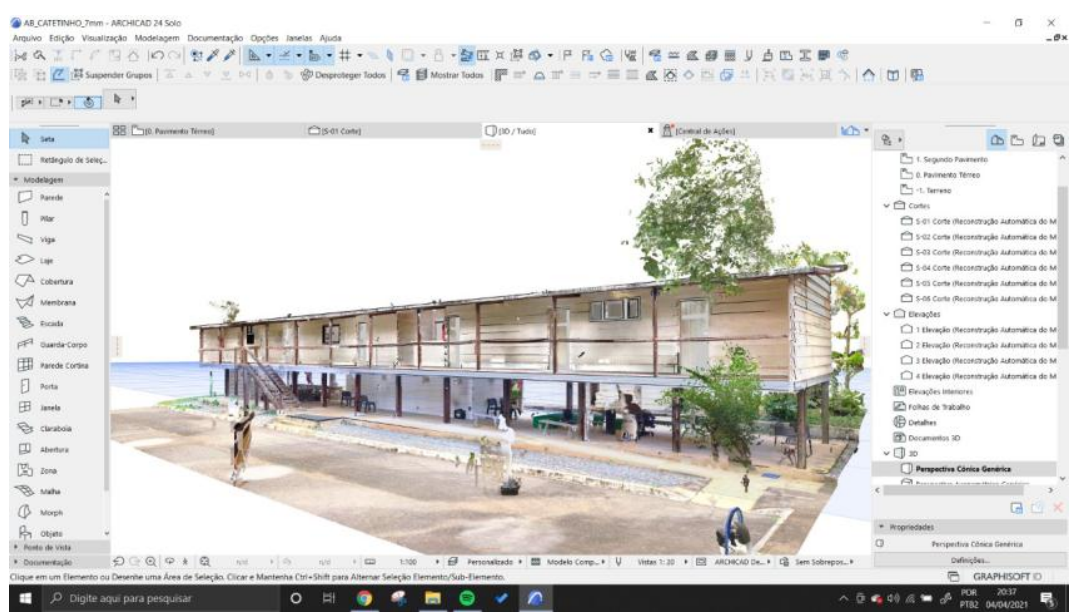

Figura 4 - Núvem de pontos Catetinho Fonte: Produzido pelo autor

Medir diretamente, modelar de modo geométrico e tridimensional, utilizar a fotogrametria digital, aplicar 3 D Laser Scanning, usar as sistemáticas informacionais geográficas e de hipermídia são os recursos tecnológicos básicos para levantar e processar informações derivadas dos acervos arquitetônicos.

\section{Metodologia}

Dentre as etapas básicas de uma metodologia de implementação HBIM, encontram-se precisamente a definição das finalidades específicas, como também a tática contratual e avaliativa do progredimento e da qualidade de serviços efetivados. A implementação implica a precisão de concordatas com empresas que trabalharão de forma colaborativa para que se realize os procedimentos no desenvolvimento de uma mesma ação. A utilização da tecnologia HBIM transforma, de maneira significativa, não apenas o modo como os trabalhos são desempenhados, mas também o modo de avaliação e medição do progresso.

O processo HBIM que será desenvolvido se constituirá a partir da edificação do Catetinho. $O$ software que será utilizado será o Archicad 2020, devido a sua versalidade, ampla utilização do mercado e aceitação vasta. O Archicad é um software que trabalha de modo integrado e geral, tendo várias funcionalidades, assim é muito requisitado. Também se usará a fotogrametria que é uma classificação da topografia topográfica local e da altimetria a partir de pares de fotografias simultâneas realizadas por duas câmeras de um distanciamento constante entre si, permitindo visão tridimensional no momento em que são analisadas por meio de aparelhamento adequado; fotocartografia.

A metodologia do presente trabalho tem como base um estudo de caso focado na modelagem do Museu do Catetinho usando ferramentas HBIM. O método proposto para o desenvolvimento do projeto se compõe de três etapas: 1 - Realização de registros no local; 2 - Colhimento de pontos chaves com a fotogrametria; 3 - Início da modelagem.
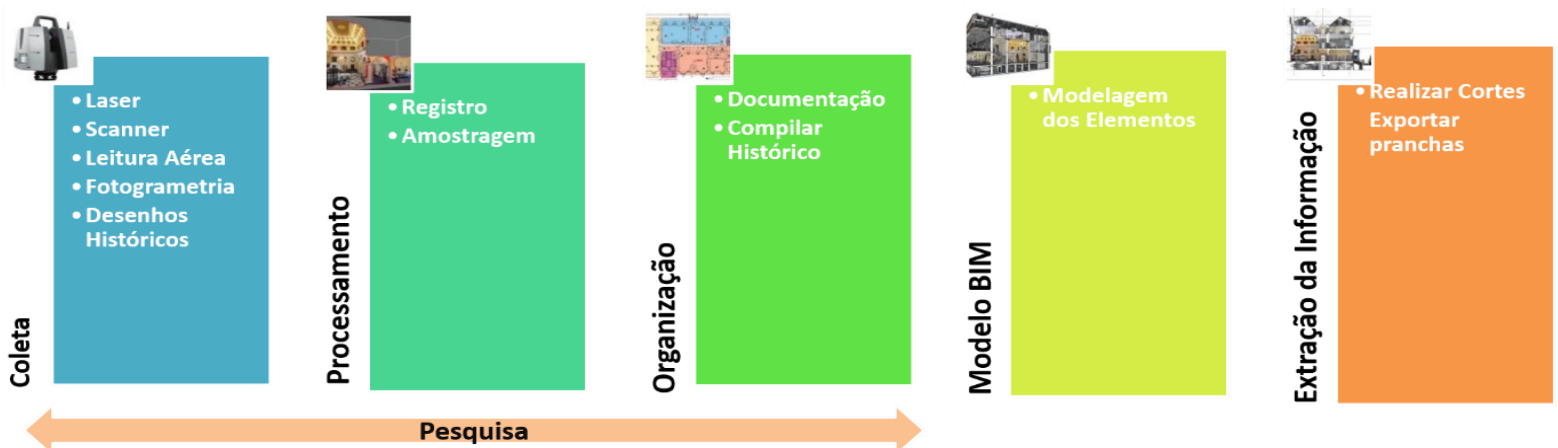

Pesquisa

Execução

Figura 5 - Processo HBIM Fonte: Produzido pelo autor 


\section{Estudo de Caso}

O palácio do Catete foi a residência oficial do então Presidente Juscelino Kubitschek, que tinha decidido transferir a capital do país a época do Rio de Janeiro para uma área no Estado de Goiás no meio do território brasileiro. A ideia de fazer uma residência provisória para o presidente. Oscar Niemeyer planejou um palácio feito de tábua, e esse viria a ser a primeira obra projetada por ele no cerrado.

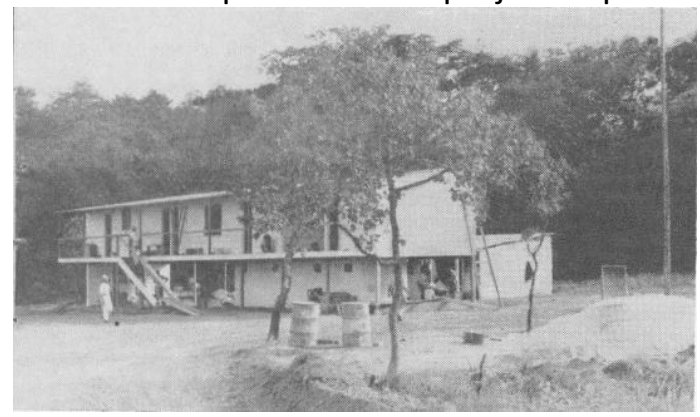

Figura 5 - Palácio do Catete em 1957 Fonte: Companhia Urbanizadora da Nova Capital do Brasil (NOVACAP)

Juscelino Kubitschek inaugura primeira obra concluída da nova capital. O catetinho foi tombado pelo patrimônio histórico a pedido do próprio JK em 1959, hoje é um museu que retrata a vida simples dos anos de construção. O catetinho é estruturado em madeira com gabinete, banheiro com água quente e fria, cozinha e muita área verde no meio do cerrado. Entre várias histórias catetinho continua elegante no meio do cerrado, banhado por água de beber, e é uma das belezas que Brasília tem além dos eixos. Considerando a importância do Catetinho em um momento singular da história e do progresso brasileiro, deu-se a escolha do museu para esta pesquisa.

Foram apresentados nesse estudo a concepção de preservação de patrimônio, a descrição do processo de documentação dos bens tombados e patrimônio histórico, a definição de cadastro e gestão de patrimônio arquitetônico por meio da tecnologia digital, o contraste com os métodos de gestão anteriores ao HBIM, o estabelecimento da diferença entre um projeto de manutenção e documentação pelo CAD e BIM, a construção e identificação das características do HBIM.

Essas constatações são imprescindíveis para que se esclareça a importância da ferramenta HBIM. Fica evidente que os benefícios de tal ferramenta ainda são pouco conhecidos, considerando também o fator de resistência para sua adesão. Entretanto, é importante pesquisas desse tipo para que metodologias mais avançadas façam parte do contexto arquitetônico, de engenharia (e de manutenção e preservação) das obras públicas.

Para o aumento da utilização dessa importante ferramenta para os profissionais que trabalham com a conservação de edificações de valor histórico e cultural, esse trabalho propõe as seguintes medidas significativas e viáveis, assim descritas:

- Realização de encontros técnicos e científicos objetivando o estabelecimento de estratégias que possam capacitar, conscientizar e sensibilizar profissionais que trabalham com a conservação de edificações tidas como patrimônio de valor histórico e cultural, da importância, utilidade e versatilidade dessa ferramenta;

- Incorporação de experiências bem sucedidas em outras nações da utilização dessa ferramenta no Brasil.

Espera-se, dessa forma, a disseminação dos benefícios da metodologia BIM, fazendo com que essa ferramenta fique conhecida e que práticas de implementação sejam desenvolvidas. Deve-se buscar fundamentar conhecimentos teóricos, abrangendo habilidades na instituição de projetos com mecanismos BIM, buscando tornar de ciência de todos a necessidade de atualizar as ferramentas de conservação do Patrimônio Histórico Cultural.

Foi iniciada a modelagem do Catetinho utilizando o Software Archicad, assim como pode ser visto nas figuras abaixo: 


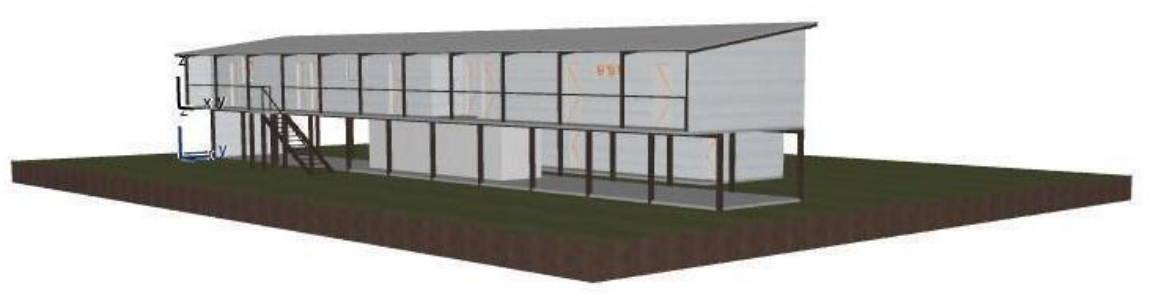

Figura 6- Perspectiva Frontal Modelagem Catetinho Fonte: Produzido pelo Autor por meio do Software Archicad

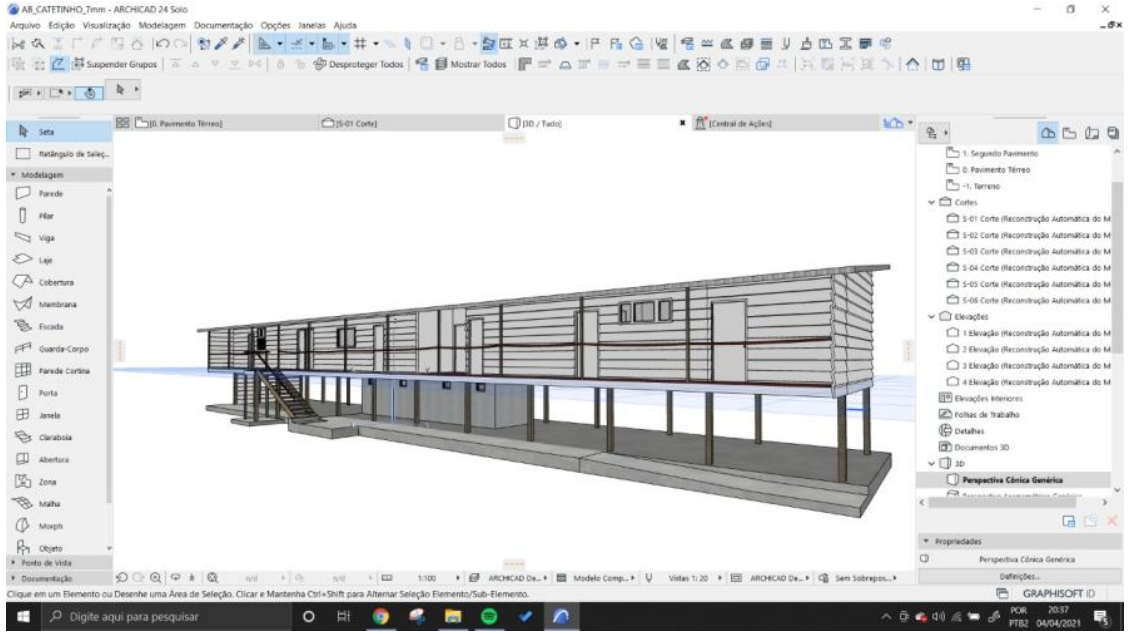

Figura 7- Modelagem Catetinho a partir da núvem de pontos Fonte: Produzido pelo Autor por meio do Software Archicad

\section{Conlcusão}

Diante do exposto, percebeu-se a importância da Modelagem da Informação da Construção (BIM) para a catalogação, salvaguarda e inventários de dados e informações relacionadas com edificações do patrimônio histórico e cultural, visando a sua conservação.

Foi feito um modelo HBIM do patrimônio artístico e histórico do Catetinho, no Distrito Federal, usando metodologia e parâmetros estabelecidos nesse trabalho, colaborando para a conservação efetiva do Patrimônio Histórico Brasileiro, visto que este é um legado que caracteriza a historicidade humana. Levando em consideração os avanços tecnológicos e a ampliação de saberes dos profissionais, faz-se necessário trabalhar de forma conjunta e eficiente objetivando "eternizar" culturas e tradições de épocas que evidenciam a realidade humana.

\section{Referências Bibliográficas}

FONSECA, M. C. L. O patrimônio em processo: trajetória da política federal de preservação no Brasil. 1 Ed. ed. [s.I.] Universidade Federal do Rio de Janeiro (UFRJ), 1997.

CUNHA, C. R., KODAIRA, T. K. O legado moderno na cidade contemporânea: restauração e uso. 8o Seminário DOCOMOMO Brasil, Rio de Janeiro, Set. 2009.

CUNHA, Cláudia dos Reis. Restauração: diálogos entre teoria e prática no Brasil nas experiências do Iphan. Tese de doutorado. Faculdade de Arquitetura e Urbanismo. Universidade de São Paulo, 2010.

JOKILEHTO, Jukka. Continuity and change in recente heritage. In: OERS, R.V.; HARAGUCHI, S. (Ed.). World Heritage papers, 5 Identification and documentation of modern heritage. Paris: UNESCO/WHC, 2003. p. 101-109.

GHIRARDELLO, N; SPISSO, B. Patrimônio histórico: como e por que preservar. Bauru: Canal 6, 2008.

COGIMA, Camila Kimi, 1994- C656b CogBIM aplicado ao patrimônio histórico baseado em levantamento híbrido com multisensores / Camila Kimi Cogima. - Limeira, SP : [s.n.], 2019. CogOrientador: Eloisa Dezen-Kempter. CogDissertação (mestrado) - Universidade Estadual de Campinas, Faculdade de Tecnologia. 
FREITAS, Laura Nascimento. Análise comparativa entre projetos de edificações utilizando métodos tradicionais e BIM [Distrito Federal] 2019. 93p. (ENC/FT/UnB, Bacharel, Engenharia Civil, 2019). Monografia de Projeto Final - Universidade de Brasília.

Câmara Brasileira da Indústria da Construção C172f Fundamentos BIM - Parte 1, 2 e 3: Implementação do BIM para Construtoras e Incorporadoras/ Câmara Brasileira da Indústria da Construção.- Brasília: CBIC, 2016. 124p.:il

GASPAR, João; LORENZO, Natália. ArchiCad: passo a passo. São Paulo: ProBooks, 2014.

DORE, C., MURPHY, M. (2012). Integration of Historic Building Information Modeling and 3D GIS for Recording and Managing Cultural Heritage Sites. 18th International Conference on Virtual Systems and Multimedia: "Virtual Systems in the Information Society", 369-376. 